\title{
Original
}

\section{Relationship between Maspin Tumor Suppressor Gene Expression and Progression of Esophageal, Gastric, and Colonic Cancer}

\author{
Nobuyuki Ohike ${ }^{1)}$, Kazuo Nagoshi ${ }^{1.2)}$, Atsushi Ishino ${ }^{1)}$, \\ Kyoko Matsukawa ${ }^{1)}$, Yuko Date ${ }^{1)}$, Kenji Iwaku ${ }^{1)}$, \\ Hiroshi IsHINO ${ }^{1)}$, Masanobu IRI ${ }^{1)}$ and Toshio MoroHOSHI ${ }^{1)}$
}

\begin{abstract}
The tumor suppressor gene maspin inhibits invasion, metastasis, and angiogenesis of cancers. The expression of maspin is reported to correlate inversely with tumor progression or prognosis in breast and prostatic cancer. We examined the relationship between maspin expression and progression of 14 surgicallyresected esophageal, 33 gastric, and 36 colonic cancer cases. Maspin expression was measured immunohistochemically and classified as high or low. In esophageal cancer cases, the frequency of high maspin expression in normal epithelium, intraepithelial cancer, invasive cancer, and lymph node metastasis were all high at $100 \%, 100 \%, 93 \%$ and $100 \%$, respectively. Whereas in cases of gastric cancer, the frequency of high maspin expression in glandular epithelium, intestinal metaplasia, early-stage cancer, advanced cancer, and lymph node metastasis was $0 \%, 100 \%, 85 \%, 89 \%$, and $91 \%$, respectively. Finally, in cases of colonic cancer, high maspin expression in normal epithelium, adenoma, early-stage cancer, advanced cancer, and lymph node metastasis was $0 \%, 100 \%, 63 \%, 70 \%$, and $90 \%$, respectively. Most squamous cell carcinomas of the esophagus showed diffuse expression of maspin, whereas gastric and colonic cancers, and especially well-differentiated adenocarcinoma, tended to show heterogenous expression patterns. There was no inverse correlation found between maspin expression and tumor progression or metastasis in gastric and colonic cancers. The high levels of maspin expression observed both in primary and metastatic lesions of the three types of cancer suggest an important role of maspin expression in these tumors and the potential use of maspin as a tumor marker. Although an association has not yet been clearly demonstrated between maspin expression and tumor progression in gastric and colonic cancers due to the heterogenous pattern of maspin expression, maspin is considered a useful prognosticator of alimentary tract cancers and is expected to be applied as a new therapeutic strategy.
\end{abstract}

Key words : tumor suppressor gene, maspin, alimentary tract cancers, immunohistochemistry

\footnotetext{
1) First Department of Pathology, Showa University School of Medicine, 1-5-8 Hatanodai, Shinagawa-ku, Tokyo 1428555 , Japan.

2) Nagoshi Clinic.
} 


\section{Introduction}

The tumor suppressor gene maspin, an inhibitor of serine proteases, inhibits invasion, metastasis, and angiogenesis of cancers ${ }^{1)}$. Studies have shown that maspin, (1) is a target gene of $\mathrm{p} 53^{2)},(2)$ is highly expressed in myoepithelia and basal cells to suppress the invasion of cancers ${ }^{3)}$, (3) interacts with $\mathrm{p} 63^{4)}$, and (4) induces apoptosis in tumor cells and in endothelial cells of tumor vasculature ${ }^{5.6)}$. Regulatory mechanisms such as DNA methylation and histone deacetylation play a suppressive role in activation of the maspin gene in various cancer cells ${ }^{7,8)}$. We have previously demonstrated similar mechanisms using pancreatic carcinoma cell lines ${ }^{9)}$. Research on maspin in tumors is expected to provide evidence for a clinical application as well as elucidating the pathophysiology of cancer. However, analyses of maspin expression in clinical cases have not always given results that reflect the tumor inhibitory role of maspin. In some reports, maspin expression decreased as the tumor progressed and was inversely correlated with poor indicators ${ }^{10.11)}$, while others reported that maspin was up-regulated along with malignant transformation or tumor progression and positively correlated with poor prognosticators ${ }^{12^{-14}}$. Conflicting results have been reported for maspin expression in the same carcinoma type ${ }^{11.13 .15-19)}$. Furthermore, there have been few studies examining the timing of maspin expression decrease during cancer progression.

Although the prognosis of early-stage cancers of the esophagus, stomach, and colon is improving due to advances in early detection and improved surgical procedures in recent years, the prognosis of advanced cancer is still poor. Understanding the molecular and biological pathophysiology of these gastrointestinal tumors is critical to an improved prognosis. Most studies investigating maspin expression in gastric and colonic cancers concluded that a decrease in maspin expression correlates with progression, metastasis, or poor prognosis of cancer ${ }^{2024)}$. By comparison, Terashima et $a l^{20)}$ reported a positive correlation between maspin expression and lymph node metastasis. To date, there have been no studies on maspin expression in esophageal cancer.

In this study, we examined the expression of maspin in a wide range of clinical materials including normal epithelia and lymph node metastases in three types of alimentary tract tumors, including esophageal, gastric, and colonic tumors, and compared expression in relation to clinicopathological characteristics.

\section{Materials and Methods}

Tissue samples were selected from surgically resected specimens at Showa University Hospital between May 2004 and April 2005 according to specimen numbers in ascending order. Fourteen cases of esophageal cancer (including 13 intraepithelial lesions), 20 earlystage and 19 advanced gastric cancer cases (including 11 cases of lymph node metastasis), and 16 early-stage and 20 advanced colonic cancer cases (including 10 cases of lymph node metastasis) were examined. One or two specimens were removed from the largest section of the tumor containing the deepest invasion and normal mucosa, and in cases with lymph node metastasis, one specimen of the lymph node metastasis was selected from the HE-stained specimens stored at the Department of Pathology. The formalin-fixed and paraffin-embedded blocks corresponding to these specimens were cut into $3 \mu \mathrm{m}$ slices for immunological staining. Immunohistochemistry using maspin monoclonal antibody (PharMingen International, San Diego, CA, U. S. A.; diluted 1:75) was performed according to the 
Table 1. Frequency of high expression of maspin from normal epithelium to cancers

\begin{tabular}{|c|c|c|c|c|c|c|}
\hline \multirow{2}{*}{ Esophagus } & Lesions & \multicolumn{2}{|c|}{ Normal epithelium } & Intraepithelial & Invasive & Lymph node \\
\hline & High expression & \multicolumn{2}{|c|}{$14 / 14(100 \%)$} & $13 / 13(100 \%)$ & $13 / 14(93 \%)$ & $10 / 10(100 \%)$ \\
\hline \multirow[t]{2}{*}{ Stomach } & Lesions & $\begin{array}{c}\text { Normal } \\
\text { epithelium }\end{array}$ & $\begin{array}{l}\text { Intestinal } \\
\text { metaplasia }\end{array}$ & Early cancer & $\begin{array}{l}\text { Advanced } \\
\text { cancer }\end{array}$ & $\begin{array}{c}\text { Lymph node } \\
\text { metastasis }\end{array}$ \\
\hline & High expression & $0 / 19(0 \%)$ & $16 / 16(100 \%)$ & $17 / 20(85 \%)$ & $17 / 19(89 \%)$ & $10 / 11(91 \%)$ \\
\hline \multirow[t]{2}{*}{ Colon } & Lesions & $\begin{array}{c}\text { Normal } \\
\text { epithelium }\end{array}$ & Adenoma & Early cancer & $\begin{array}{l}\text { Advanced } \\
\text { cancer }\end{array}$ & $\begin{array}{c}\text { Lymph node } \\
\text { metastasis }\end{array}$ \\
\hline & High expression & $0 / 16(0 \%)$ & $5 / 5(100 \%)$ & $10 / 16(63 \%)$ & $14 / 20(70 \%)$ & $9 / 10(90 \%)$ \\
\hline
\end{tabular}

EnVision ChemMate method (details are provided in Ref. ${ }^{16)}$ ). Briefly, the antigen retrieval method requires pretreating sections by microwaving for $10 \mathrm{~min}$ in $10 \mathrm{mM}(\mathrm{pH}$ 6) citrate buffer and incubating with anti-human maspin monoclonal antibody for $30 \mathrm{~min}$ followed by incubation with HRP-conjugated dextran polymer reagent (DakoCytomation) for 30 min. The chromogen was 3'3-Diaminobenzidine. Normal mammary gland tissues were used as positive controls. Moderate to high staining of tumor cells occupying more than $30 \%$ of the tumor was considered to represent high maspin expression ${ }^{9)}$. Statistical analysis of maspin expression and clinicopathological factors was performed using t-test and chi-square test, and $\mathrm{p}<0.05$ was considered statistically significant.

\section{Results}

\section{Progression of various cancers and maspin expression}

In the esophagus, all normal squamous epithelia and intraepithelial cancers showed high maspin expression (Table 1). Most invasive cancers also showed high expression, with the exception of one case. All cases of lymph nodes metastases also showed high maspin expression. Cancer cells showed strong expression in either the cytoplasm, or in both the cytoplasm and nucleus, whereas normal epithelial cells showed relatively weak staining in the cytoplasm and maspin expression tended to localize in the nucleus (Fig. 1-1).

In the stomach, all intestinal metaplastic epithelia showed a high level of expression in contrast to the low expression observed in normal gastric glandular epithelia (Table 1). Both early-stage and advanced cancers frequently showed high maspin expression. High maspin expression was more prevalent in metastatic lymph nodes.

In the colon, normal epithelia showed low maspin expression, while epithelia adjacent to the tumor showed a tendency toward strong expression (Fig. 3-1). All adenomas showed a high level of maspin expression (Table 1). Although less frequently than gastric cancers, both early-stage and advanced colonic cancers showed a high level of maspin expression in more than $50 \%$ of cases. Lymph node metastases showed a high expression similar to esophageal and gastric cancers.

\section{Expression pattern of maspin (diffuse or heterogenous)}

During this study, we found that the maspin expression pattern was diffuse and heterogeneous (Figs. 1-2, 2-1, 3-2) under various conditions. In the esophagus, all normal epithelia, 
$1-1$

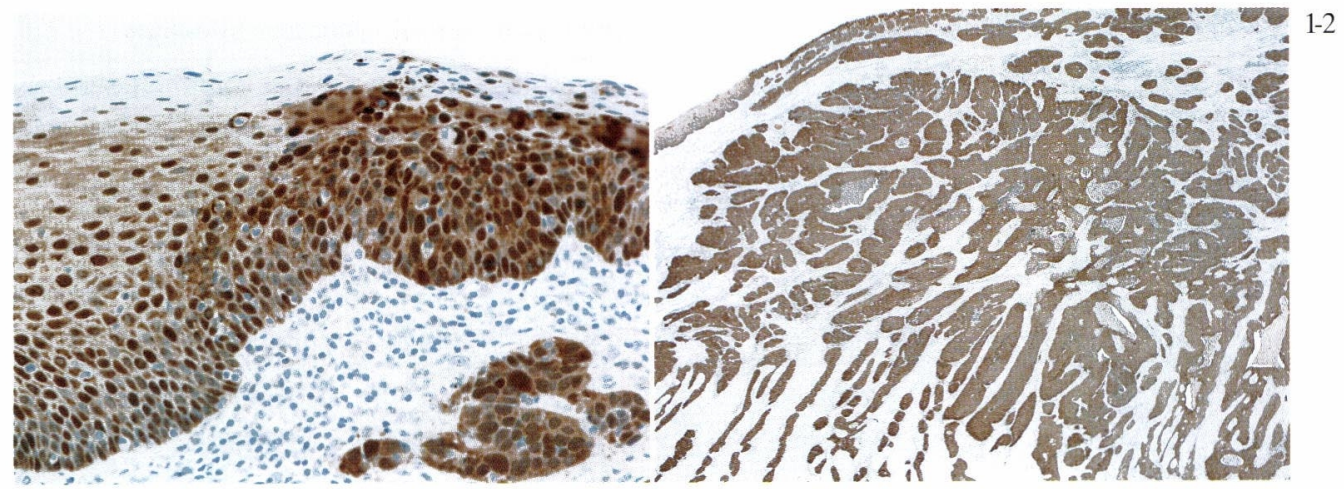

Fig. 1-1. Maspin expression is somewhat enhanced from normal epithelium (left side) to carcinoma in situ and invasive small nests (right side) of the esophagus.

2. The invasive esophageal cancer shows a diffuse expression of maspin throughout the tumor.

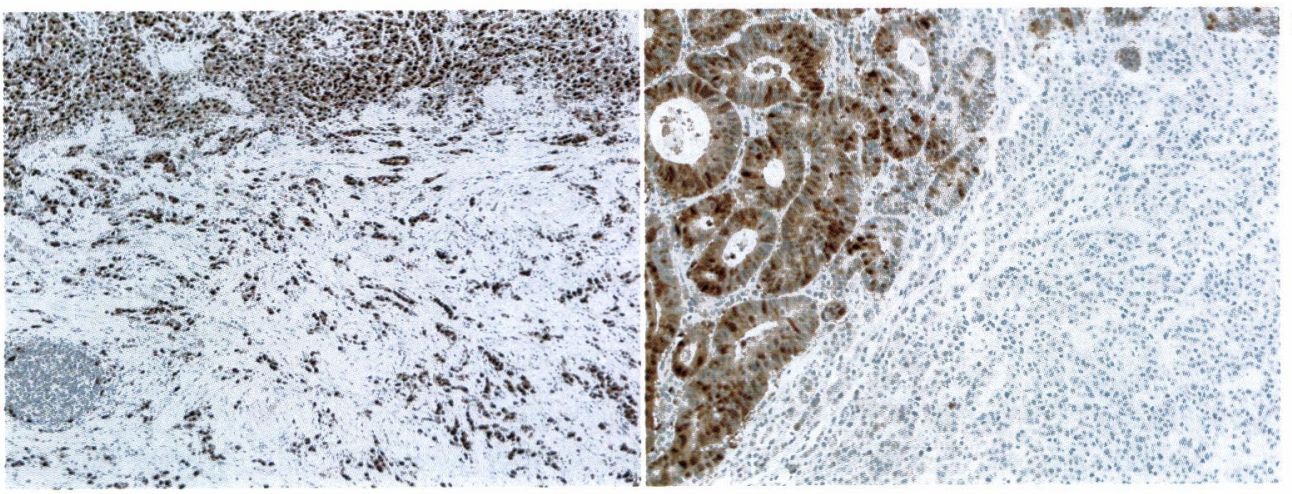

Fig. 2-1. The poorly differentiated gastric adenocarcinoma shows a scirrhous invasion and a diffuse expression of maspin.

2. Maspin expression is reduced in the undifferentiated carcinoma area (right side) in contrast to a high expression of maspin in tubular adenocarcinoma area (left side).
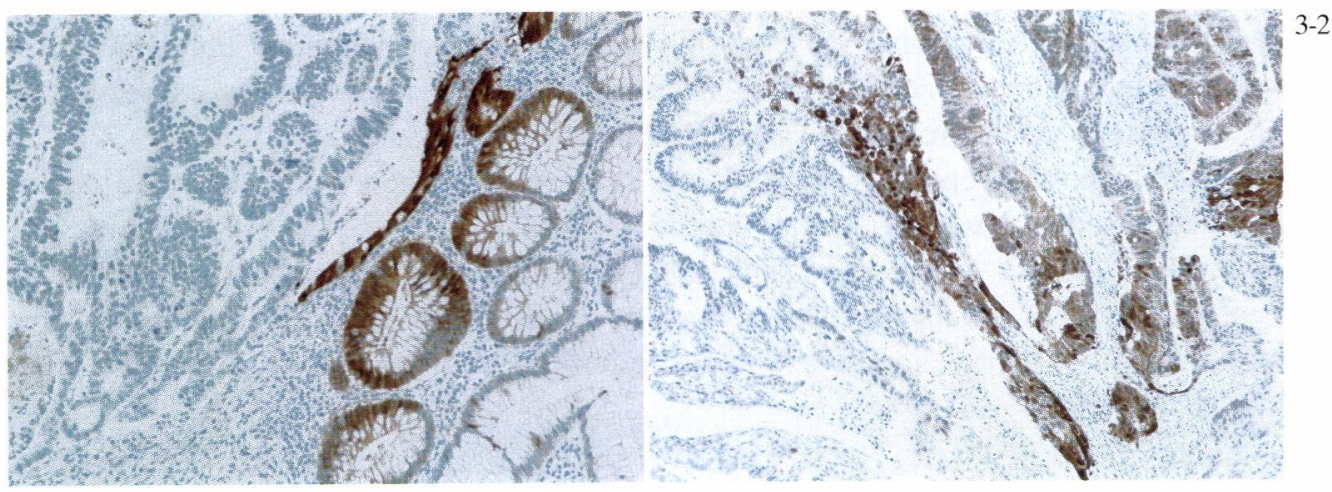

Fig. 3-1. Maspin expression is observed in colonic epithelium (right side) adjacent to cancer (left side) that lacks maspin expression.

2. The well-differentiated colonic adenocarcinoma shows a heterogeneous expression of maspin. 
Table 2. Frequency of heterogenous expression (vs diffuse) of maspin in various cancers

\begin{tabular}{lcccc}
\hline & & Esophageal cancer & Gastric cancer & Colonic cancer \\
Total & & $2 / 14(14 \%)$ & $11 / 39(28 \%)$ & $27 / 36(75 \%)$ \\
Histological grade & well to moderate & $1 / 10$ & $8 / 18^{*}$ & $27 / 35$ \\
& poorly or others & $1 / 4$ & $3 / 21^{*}$ & $0 / 1$ \\
\hline
\end{tabular}

${ }^{*} \mathrm{p}=0.04$

intraepithelial cancers, and lymph node metastases showed diffuse maspin expression. Although most invasive cancers also showed diffuse expression on the whole (Fig. 1-2), heterogenous expression was observed in two cases (one case of low expression and one case of high expression) (Table 2). The former had diffuse and high maspin expression in the lymph node metastases. The latter was the only case with a partly sarcomatous change in which the expression of maspin decreased.

In the stomach, all normal glandular epithelia showed heterogenous maspin expression, while all intestinal metaplastic epithelia had diffuse expression patterns. In the cancer cases, 11 cases (28\%; five cases with low maspin expression and six cases with high expression) showed heterogenous patterns of maspin expression. Heterogenous expression was observed in relatively well-differentiated tubular adenocarcinoma, whereas scirrhous, poorly-differentiated adenocarcinoma and signet ring cell carcinoma showed primarily diffuse expression (Table 2, Fig. 2-1). In addition, in one case an extremely low maspin expression was seen in the highly dedifferentiated component of undifferentiated carcinoma (Fig. 2-2).

In the colon, all normal epithelia showed heterogenous maspin expression patterns, whereas all adenomas showed diffuse expression. In the cancer cases, both early stage (13/16) and advanced (14/20) cases frequently showed heterogenous maspin expression (Table 2), while a few could not be classified as having either low or high maspin expression (Fig. 3-2).

\section{Statistical analysis of maspin expression and clinicopathological factors}

The relationship between maspin expression and clinicopathological factors was determined in patients with gastric cancers or colonic cancers (Tables 3 and 4). In gastric cancer low maspin expression was more frequent in males and in well-differentiated tumors, although the difference did not reach statistical significance due to the small sample size. There was no significant correlation between maspin expression and the depth of tumor invasion or the presence of lymph node metastasis. Similarly in colonic cancer, there was no significant correlation between maspin expression and various clinicopathological characteristics. However, lymph node metastasis was more frequent in cases with high expression of maspin.

\section{Discussion}

This study demonstrated a high prevalence of elevated maspin expression in a wide range of esophageal tissues including normal epithelium, invasive carcinoma, and lymph node metastasis. The strong relationship of squamous epithelial cells and maspin expression has already been found in oral mucosa, skin, epithelium of cervix, and squamous metaplasia in the epithelium of pancreatic duct and bronchus ${ }^{12,16.25)}$. The finding of high maspin expression in 13 of 14 invasive cancers in the study suggests a close involvement of maspin in 
Table 3. Comparison of clinicopathological features between maspin high- and low-expression groups in gastric cancer

\begin{tabular}{|c|c|c|c|}
\hline Maspin expression & High & Low & $p$-value \\
\hline $\mathrm{n}$ & 34 & 5 & \\
\hline Average age (years) & 65.4 & 65.8 & NS \\
\hline Gender (male : female) & $20: 14$ & $4: 1$ & NS \\
\hline Histological grade, well-mderate & $14 / 34$ & $4 / 5$ & NS \\
\hline Advanced stage (depth; mp, ss, se) & $16 / 34$ & $3 / 5$ & NS \\
\hline Lymph node metastasis & $9 / 34$ & $2 / 5$ & NS \\
\hline \multirow{2}{*}{$\begin{array}{l}\text { Stage } I(n=26) \\
\text { Stage } \operatorname{II}-\operatorname{IV}(n=13)\end{array}$} & $23 / 26$ & $3 / 26$ & \multirow{2}{*}{ NS } \\
\hline & $11 / 13$ & $2 / 13$ & \\
\hline
\end{tabular}

NS : not significant

Table 4. Comparison of clinicopathological features between maspin high- and low-expression groups in colonic cancer

\begin{tabular}{|c|c|c|c|}
\hline Maspin expression & High & Low & $p$-value \\
\hline $\mathrm{n}$ & 24 & 12 & \\
\hline Average age (years) & 65.8 & 70.8 & NS \\
\hline Gender (male : female) & $14: 10$ & $7: 5$ & NS \\
\hline Histological grade, well-moderate & $23 / 24$ & $12 / 12$ & NS \\
\hline Advanced stage (depth; mp, ss, se) & $14 / 24$ & $6 / 12$ & NS \\
\hline Lymph node metastasis & $8 / 24$ & $2 / 12$ & NS \\
\hline Stage grouping $\begin{array}{l}\text { Stage } 0-I(n=23) \\
\text { Stage } \operatorname{II}-\operatorname{IV}(n=13)\end{array}$ & $\begin{array}{l}14 / 23 \\
10 / 13\end{array}$ & $\begin{array}{l}9 / 23 \\
3 / 13\end{array}$ & NS \\
\hline
\end{tabular}

NS : not significant

tumor growth. Others reported that maspin was down-regulated with tumor progression and patients with low maspin expression had a poor prognosis in cervical ${ }^{26)}$ and oral ${ }^{27,28)}$ cancer cases, which have as high an incidence of squamous cell carcinoma as esophageal cancer. Although a similar association is expected in esophageal cancer, we could not perform a statistical comparison of maspin expression and clinicopathological characteristics as there was only one case with a low maspin expression. Further investigations with a larger patient population are necessary.

The stomach and colon both have glandular epithelium and we found similar maspin expression levels in both. While normal epithelia in both organs showed low maspin expression, all intestinal metaplasia of the stomach and adenomas of the colon showed diffuse and high expression. To support this finding from the molecular level, Akiyama et al demonstrated that the maspin gene promoter region in gastric glandular epithelium was hyper-methylated, whereas in epithelium with intestinal metaplasia, the maspin gene promoter was hypomethylated ${ }^{29)}$. We also found normal epithelium had a tendency toward higher maspin expression around the tumor. These findings appear to imply a strong inhibitory effect against malignant transformation or invasion of carcinoma. Although both gastric and colonic cancers frequently showed high maspin expression irrespective of progression, colonic cancers had more cases of low expression. One reason for this difference is that 
most colonic cancers were differentiated, while $50 \%$ of the gastric cancer cases were differentiated and the rest were poorly differentiated including scirrhous carcinoma and signet ring cell carcinoma. Because differentiated cancers tended to show heterogenous maspin expression covering less than one-third of the tumor, more colonic cancers were classified as low expression. This heterogenous expression may be the reason for the contradictory findings. Other studies have shown either a positive or a reverse correlation between maspin expression and tumor progression or the prognosis in the same tumor types, particularly in the lung ${ }^{15.16)}$, stomach ${ }^{20-22)}$, thyroid ${ }^{13.17)}$, and mammary gland ${ }^{11.18 .19)}$ where adenocarcinoma is the dominant type. Therefore this study shows that maspin expression was not significantly correlated with cancer progression and that lymph node metastases were more frequent in cases with high maspin expression rather than low expression, but does not deny the tumor inhibitory function of maspin in cancer cells.

In this study, maspin expression in lymph node metastases was similar to or higher than in the primary lesion contrary to our expectations. A previous study showed elevated maspin expression in lymphocyte-rich stroma ${ }^{30)}$. Further determination of maspin expression at the metastatic level other than lymph nodes is necessary. In gastric tumors, poorly differentiated carcinoma showed higher expression, whereas extremely low expression of maspin was observed in the undifferentiated component of highly dedifferentiated gastric carcinoma and sarcomatoid component of esophagus. These findings illustrate the association of maspin with the degree of malignancy and the close relation with epithelial differentiation, and suggest a broad role for maspin in cancer.

\section{Conclusion}

The high incidence of maspin expression observed in both primary and metastatic lesions of esophageal, gastric, and colonic cancer suggested an important role for maspin expression in these tumors and the potential use of maspin as a tumor marker. The confusing relationship between maspin expression and tumor progression or prognosis in adenocarcinoma is probably due to the heterogenous expression of maspin frequently seen in welldifferentiated adenocarcinoma.

\section{References}

1) Zou Z, Anisowicz A, Hendrix MJ, Thor A, Neveu M, Sheng S, Rafidi K, Seftor E and Sager R: Maspin, a serpin with tumor-suppressing activity in human mammary epithelial cells. Science 263:526-529 (1994)

2) Zhang $W$ and Zhang $M$ : Tissue microarray analysis of maspin expression and its reverse correlation with mutant p53 in various tumors. Int J Oncol $20: 1145-1150$ (2002)

3) Sternlicht MD, Kedeshian P, Shao ZM, Safarians S and Barsky SH: The human myoepithelial cell is a natural tumor suppressor. Clin Cancer Res $3: 1949-1958$ (1997)

4) Kim S, Han J, Kim J and Park C: Maspin expression is transactivated by p63 and is critical for the modulation of lung cancer progression. Cancer Res $64: 6900-6905$ (2004)

5) Jiang N, Meng Y, Zhang S, Mensah-Osman E and Sheng S: Maspin sensitizes breast carcinoma cells to induced apoptosis. Oncogene $21:$ 4089-4098 (2002)

6) Li Z, Shi HY and Zhang M: Targeted expression of maspin in tumor vasculatures induces endothelial cell apoptosis. Oncogene 24:2008-2019 (2005)

7) Maass N, Biallek M, Rosel F, Schem C, Ohike N, Zhang M, Jonat W and Nagasaki K: Hypermethylation and histone deacetylation lead to silencing of the maspin gene in human breast cancer. Biochem Biophys Res Commun 297 : 125-128 (2002)

8) Sugimoto S, Maass N, Takimoto Y, Sato K, Minei S, Zhang M, Hoshikawa Y, Junemann KP, Jonat W and Nagasaki K: Expression and regulation of tumor suppressor gene maspin in human bladder cancer. Cancer Lett 203 : 209-215 (2004) 
9) Ohike N, Maass N, Mundhenke C, Biallek M, Zhang M, Jonat W, Luttges J, Morohoshi T, Kloppel G and Nagasaki K : Clinicopathological significance and molecular regulation of maspin expression in ductal adenocarcinoma of the pancreas. Cancer Lett 199: 193-200 (2003)

10) Machtens S, Serth J, Bokemeyer C, Bathke W, Minssen A, Kollmannsberger C, Hartmann J, Knuchel R, Kondo $\mathrm{M}$, Jonas $\mathrm{U}$ and Kuczyk $\mathrm{M}$ : Expression of the p53 and Maspin protein in primary prostate cancer: correlation with clinical features. Int $J$ Cancer $95: 337-342$ (2001)

11) Maass N. Hojo T, Rosel F, Ikeda T, Jonat W and Nagasaki K: Down regulation of the tumor suppressor gene maspin in breast carcinoma is associated with a higher risk of distant metastasis. Clin Biochem 34:303-307 (2001)

12) Maass N, Hojo T, Ueding M, Luttges J, Kloppel G, Jonat W and Nagasaki K: Expression of the tumor suppressor gene Maspin in human pancreatic cancers. Clin Cancer Res $7: 812-817$ (2001)

13) Ito Y, Yoshida H, Tomoda C, Uruno T, Takamura Y, Miya A, Kobayashi K, Matsuzuka F, Matsuura N, Kuma $\mathrm{K}$ and Miyauchi A : Maspin expression is directly associated with biological aggressiveness of thyroid carcinoma. Thyroid 14 : 13-18 (2004)

14) Sood AK, Fletcher MS, Gruman LM, Coffin JE, Jabbari S, Khalkhali-Ellis Z, Arbour N, Seftor EA and Hendrix MJ : The paradoxical expression of maspin in ovarian carcinoma. Clin Cancer Res 8:2924-2932 (2002)

15) Hirai K, Koizumi K, Haraguchi S, Hirata T, Mikami I, Fukushima M, Yamagishi S, Kawashima T, Okada D, Shimizu $\mathrm{K}$ and Kawamoto $\mathrm{M}$ : Prognostic significance of the tumor suppressor gene maspin in non-small cell lung cancer. Ann Thorac Surg $79: 248-253$ (2005)

16) Nakashima M, Ohike N, Nagasaki K, Adachi $M$ and Morohoshi $T$ : Prognostic significance of the maspin tumor suppressor gene in pulmonary adenocarcinoma. J Cancer Res Clin Oncol 130 : 475-479 (2004)

17) Boltze C. Schneider-Stock R, Meyer F, Peters B, Quednow C, Hoang-Vu C and Roessner A : Maspin in thyroid cancer: its relationship with p53 and clinical outcome. Oncol Rep 10:1783-1787 (2003)

18) Umekita Y, Ohi Y, Sagara Y and Yoshida H: Expression of maspin predicts poor prognosis in breast-cancer patients. Int J Cancer 100 : 452-455 (2002)

19) Maass N, Teffner M, Rosel F, Pawaresch R, Jonat W, Nagasaki K and Rudolph P: Decline in the expression of the serine proteinase inhibitor maspin is associated with tumour progression in ductal carcinomas of the breast. J Pathol 195 : 321-326 (2001)

20) Terashima M, Maesawa C, Oyama K, Ohtani S, Akiyama Y, Ogasawara S, Takagane A. Saito K, Masuda T, Kanzaki N. Matsuyama S, Hoshino Y, Kogure M, Gotoh M, Shirane M and Mori K: Gene expression profiles in human gastric cancer: expression of maspin correlates with lymph node metastasis. Br J Cancer $92: 1130^{-}$ 1136 (2005)

21) Wang MC, Yang YM, Li XH, Dong F and Li Y: Maspin expression and its clinicopathological significance in tumorigenesis and progression of gastric cancer. World J Gastroenterol 10:634-637 (2004)

22) Ito R, Nakayama $\mathrm{H}$, Yoshida $\mathrm{K}$. Oda $\mathrm{N}$ and Yasui $\mathrm{W}$ : Loss of maspin expression is associated with development and progression of gastric carcinoma with p53 abnormality. Oncol Rep 12 : 985-990 (2004)

23) Boltze C: Loss of maspin is a helpful prognosticator in colorectal cancer: a tissue microarray analysis. Pathol Res Pract 200 : 783-790 (2005)

24) Song SY, Lee SK, Kim DH, Son HJ, Kim HJ, Lim YJ, Lee WY, Chun HK and Rhee JC: Expression of maspin in colon cancers: its relationship with p53 expression and microvessel density. Dig Dis Sci 47:18311835 (2002)

25) Marioni G, Blandamura S, Giacomelli L, Calgaro N, Segato P, Leo G, Fischetto D, Staffieri A and de Filippis $\mathrm{C}$ : Nuclear expression of maspin is associated with a lower recurrence rate and a longer disease-free interval after surgery for squamous cell carcinoma of the larynx. Histopathology 46:576-582 (2005)

26) Xu C, Qudds MR, Sung CJ Steinhoff MZ, Zhang C and Lawrence WD: Maspin expression in CIN 3, microinvasive squamous cell carcinoma, and invasive squamous cell carcinoma of the uterine cervix. Mod Pathol (2005) (in press)

27) Yasumatsu R, Nakashima T, Hirakawa N, Kumamoto $Y$, Kuratomi Y, Tomita $\mathrm{K}$ and Komiyama S: Maspin expression in stage I and II oral tongue squamous cell carcinoma. Head Neck 23 : 962-966 (2001)

28) Xia W, Lau YK, Hu MC, Li L, Johnston DA, Sheng S, El-Naggar A and Hung MC: High tumoral maspin expression is associated with improved survival of patients with oral squamous cell carcinoma. Oncogene 19: 2398-2403 (2000)

29) Akiyama Y, Maesawa C, Ogasawara S, Terashima M and Masuda T: Cell-type-specific repression of the maspin gene is disrupted frequently by demethylation at the promoter region in gastric intestinal metaplasia and cancer cells. Am J Pathol 163 : 1911-1919 (2003)

30) Kim DH, Yoon DS, Dooley WC, Nam ES, Ryu JW, Jung KC, Park HR, Sohn JH, Shin HS and Park YE: Association of maspin expression with the high histological grade and lymphocyte-rich stroma in early-stage breast cancer. Histopathology $42: 37-42$ (2003) 